Annales de Parasitologie, Paris, t. 40, 1965, $\mathrm{n}^{\circ} 1$, pp. 51 à 54

\title{
Importance des Léporidés comme réservoirs sauvages dans l'épidémiologie des distomatoses à Fasciola bepatuca et Dicrocoelium dendriticum
}

\author{
par J. BAILENGER, J. TRIBOULEY, B. AMYOT et J. DURET
}

L'ubiquité de Fasciola hepatica et de Dicrocoelium dendriticum doit être considérée pour comprendre leur épidémiologie et les difficultés de leur prophylaxie.

Leur parasitisme ne s'étend pas seulement à l'homme et aux animaux domestiques, mais affecte aussi les animaux sauvages. Granville (2), Martel (5), Mickay (6), Olsen (9), Pillers (11), Simintzis (13), et Thomas (14), entre autres, ont attiré l'attention sur l'intervention des Léporidés comme réservoirs parasitaires. Ils insistent sur la nécessité de considérer le rôle des Léporidés dans la dissémination des Douves hépatiques aux Ruminants domestiques et pour Thomas l'extermination de ces parasites sera impossible tant que les lapins et les lièvres seront présents dans la zone infectée.

Nous avons voulu nous rendre compte de la fréquence du parasitisme des Léporidés par Fasciola hepatica et Dicrocoelium denditricum dans cette région des Basses-Pyrénées où les travaux poursuivis depuis plus de dix ans nous ont fourni l'occasion d'insister sur l'importance et la continuité de l'endémie distomienne : de 1957 à 1959, nous avons relevé 107 cas de distomatose diagnostiqués chirurgicalement, ou par l'examen coprologique et immunologique $(1,10)$. En 1959 , le pourcentage des moutons atteints était évalué aux environs de 50.

Ce mémoire se propose de présenter les premiers résultats d'une enquête en cours et portant essentiellement sur des lièvres (Lepus europeus) et des lapins de garenne (Oryctolagus cuniculus). Quelques Isards (Rupicapra pyrenaica) et Bouquetins (Capra ibex) pyrénéens y sont également inclus.

Le parasitisme de tous ces animaux a été décelé par l'analyse des excréments prélevés à l'autopsie. L'expérience nous a montré que la technique la plus efficace en même temps que la plus rapide pour rechercher les œufs de la grande Douve et ceux de la petite Douve hépatiques est celle décrite par Janeckso et Urbanyi et introduite en France par Roman et Euzéby (12). C'est une méthode de concentration par flottage qui utilise une solution d'iodomercurate de potassium concentrée et de densité élevée $(\mathrm{De}=1,440)$. 
Les résultats que nous avons obtenus dans ces conditions font l'objet du tableau I qu'il convient de compléter en faisant mention des résultats fournis par l'analyse des excréments de quatre Caprins sauvages tués dans la montagne avoisinante : trois Isards et un Bouquetin. Les selles de ces animaux ne contenaient pas d'œufs caractéristiques de Douves. En outre, il convient de préciser que chez six lapins, soit $11 \%$ des rongeurs parasités, les deux espèces de Douves étaient simultanément présentes.

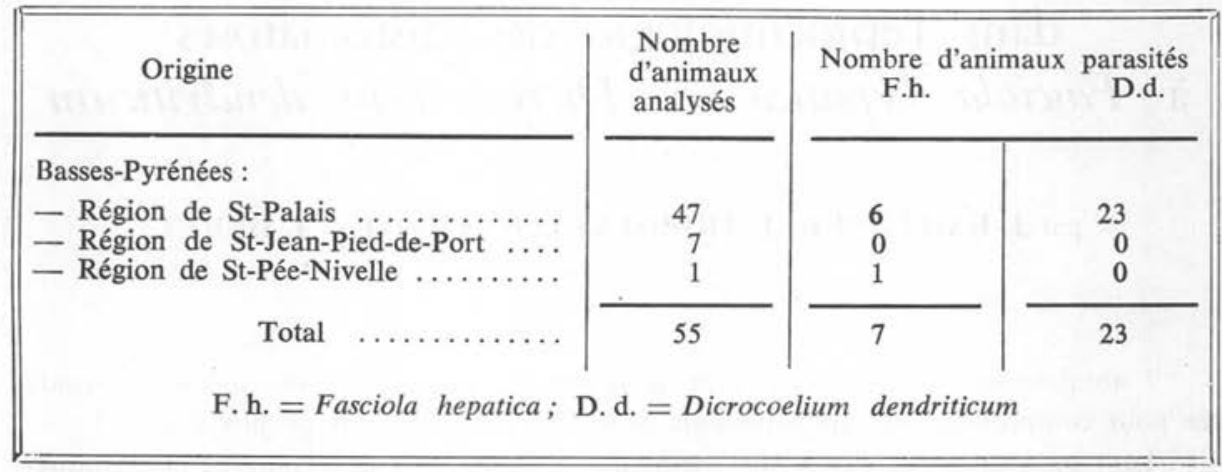

TABleau I. - Fréquence du Parasitisme des Léporidés sauvages

L'analyse du tableau démontre la fréquence de ces deux Douves chez les Léporidés de la région des Basses-Pyrénées.

Cet important pourcentage s'accompagne d'un intense parasitisme à en juger d'après le nombre d'œufs décelés à l'examen coprologique et plus encore d'après la dissection du foie d'un lapin de garenne, des canalicules biliaires duquel nous avons isolé $26 \mathrm{~F}$. hepatica *.

Ce nombre confirme les observations de Pillers (11) qui isole 22, 33 et 33 adultes identiques à $F$. hepatica de trois lapins recueillis dans une région d'Angleterre où les moutons n'avaient pas accès depuis plus de sept ans. Thomas (14), en 1883, isole 40 à $50 \mathrm{~F}$. hepatica de lapins capturés au voisinage d'Oxford.

Les infestations expérimentales effectuées avec Fasciola, permettent de classer le lapin comme un hôte de choix pour ce parasite, tant en ce qui concerne l'aptitude à s'infester que la durée du parasitisme. Montgomérie (8) isole 4, 7 et 11 Douves de trois lapins respectivement infestés par 6, 15 et 40 cercaires; Kerr et Pétkovitch (4), en infestant trois lapins par 13 cercaires, obtiennent 5, 9 et 11 Douves ; Urquhart (15), en travaillant sur trente-deux lapins à chacun desquels il donne 50 cercaires, isole du foie de chaque animal un nombre moyen de 18,6 Fasciola avec des extrêmes allant de 4 à 35. C'est-à-dire que $37,2 \%$ des 1.600 cercaires mises en expériences se sont développées. Ce pourcentage est celui auquel parvient Montgomérie dans ses expériences d'infestation expérimentale du mouton $(37,4 \%)$. Quant à la durée du parasitisme, nous ne connaissons pas d'expérience qui ait suivi son évolution jusqu’à déparasitage spontané, mais Montgomérie a pratiqué une autopsie 37 mois après le repas infestant qui avait comporté 40 cercaires et a isolé 11 Douves.

* Ce foie nous a été remis par le Docteur Berlureau, Médecin vétérinaire, Directeur des Abattoirs municipaux, que nous remercions tout particulièrement. 
Quant à D. dendriticum, Hohorst et Lämmler (3), qui en ont particulièrement étudié l'infestation expérimentale, notent qu'à partir d'une dose infestante de 100 métacercaires tous les lapins sont contaminés et que pour 1.000 métacercaires, 25 à $40 \%$ se développent à l'état adulte.

Nous avons voulu apprécier la durée du parasitisme chez ces Rongeurs. Dans ce but, nous avons infesté deux lapins respectivement par 150 et 300 métacercaires isolées de fourmis (Formica rufibarbis) *. Six mois plus tard, leurs selles renferment encore des œufs de Dicrocoelium. L'un d'eux (celui ayant reçu 300 métacercaires) est mort à cette époque par suite d'une épidémie sévissant dans l'élevage, l'autre est encore infesté au bout de vingt-trois mois. L'expérimentation se poursuit.

Il convient de noter que le parasitisme ne semble pas être toujours parfaitement toléré par l'hôte à en juger par la description que certains vétérinaires font d'épizooties rapportées presque toujours à $F$. hepatica et qui ont pour symptômes une cachexie et une anémie intenses accompagnées d'épanchements et d'œdèmes chez des animaux dont le foie sclérosé présente des canaux biliaires à parois fibreuses.

\section{Conclusions}

Nous apportons, par le travail en cours, des preuves supplémentaires affirmant l'intensité de l'endémie distomienne à Fasciola hepatica et Dicrocoelium dendriticum dans la zone des Pyrénées-Atlantiques, en attirant l'attention sur un fait bien connu, mais jusqu'ici non signalé dans cette région : 23 des 55 Léporidés sauvages (lièvres et lapins de garenne) étudiés sont porteurs de Dicrocoelium dendriticum et sept sont parasités par Fasciola hepatica. Six de ces derniers hébergent simultanément $D$. dendriticum.

L'intensité du parasitisme semble élevée : 26 grandes Douves ont été retirées du foie d'un lapin.

Sa durée semble également pouvoir se prolonger: trente-sept mois au minimum pour $F$. hepatica dans l'expérience de Montgomérie, vingt-trois mois au minimun pour D. dendriticum dans une expérimentation en cours.

La fréquence de ce parasitisme jointe à son intensité et à sa durée que confirment les distomatoses expérimentalement réalisées chez le lapin, font de ces Rongeurs un réservoir dont le rôle épidémiologique mérite de retenir l'attention.

\section{Bibliographie}

1. Bailenger J. et Pautrizel R., 1960. - La Distomatose, I. Etat actuel de son épidémio. logie dans le Sud-Ouest de la France. Rev. Hyg. et Méd. soc., 8, 603-617.

2. Granville A., Godbille M. et Grégoire C., 1952. - Distomatose chez les lapins sauvages. Ann. Méd. Vét., 96, 187.

* Les fourmis parasitées nous ont été aimablement adressées par le Dr. Hohorst, Institut de Parasitologie des Laboratoires Farbwerke Hoechst., Frankfurt am Main. 
3. Hohorst (W.) et Lammler (G.), 1962. - Experimentelle Dicrocoeliose Studien. Zeit. $f$. Tropenmed. u. Paras., 13, 377-397.

4. Kerr K. B. et Petrovitch O. L., 1935. - Active immunity in rabbits to the liver fluke Fasciola hepatica. J. Parasitol., 21, 319-320.

5. MARTel H., 1908. - Rapport sur les opérations du service vétérinaire de Paris et du département de la Seine pendant l'année 1908.

6. Mickay A. C., 1925. - Fasciola hepatica in rabbits. J. Austral. Vet. Assoc., 1, 66.

7. Montgomerie R. F., 1928. - Observations on artificial infestation of sheep with Fasciola hepatica and on a phase in the development of the parasite. J. Helminthol., 6, 167 174.

8. - 1931. - On the longevity of Fasciola hepatica in experimentaly infected rabbits. $J$. Helminthol., 9, 202-212.

9. Olsen O. X., 1948. - Wild Rabbits as reservoir hosts of the common liver fluke, Fasciola hepatica, in Southern Texas. J. Parasit., 34, 119-123.

10. Pautrizel R., Bailenger J. et Caillau M., 1960. - La Distomatose. II. Diagnostic sérologique. Rev. d'Hyg. et de Méd. soc., 8, 618-631.

11. Pillers A. N., 1926. - Fasciola hepatica in the Wild Rabbit in England. Ann. Trop. Med. et Parasit., 20, 219.

12. Roman E. et Euzeby J., 1956. - Recherche des œufs de grande Douve dans les matières fécales humaines. Bull. Assoc. Dipl. de Microb. Fac. Pharm. de Nancy, 64.

13. Simintzis G., 1951. - Distomatose des Léporidés sauvages dans les Dombes. Forte mortalité des Léporidés sauvages due à Fasciola hepatica. Bull. Soc. Sc. Vet., Lyon, 4, 136.

14. Тномаs A. P., 1883. - The natural history of the liver fluke and the prévention of rot. J. Roy. Agr. Soc. Eng., 19, 276-305.

15. Urquhart G. M., 1954. - The rabbit as host in experimental Fasciolasis. Exp. Paras., $3,38-44$.

(Laboratoire d'Immunologie et Biologie parasitaire de la Faculté de Médecine et de Pharmacie de Bordeaux)

(Professeur Pautrizel) 